\title{
The outer limiting membrane (OLM) revisited: clinical implications
}

This article was published in the following Dove Press journal:

Clinical Ophthalmology

27 March 2010

Number of times this article has been viewed

\author{
S Omri ${ }^{1,2,3}$ \\ B Omri $i^{1,2,3}$ \\ M Savoldelli ${ }^{1,2,3,4}$ \\ $L$ Jonet ${ }^{1,2,3}$ \\ B Thillaye-Goldenberg ${ }^{1,2,3}$ \\ G Thuret Th $^{5}$ \\ P Gain ${ }^{5}$ \\ J C Jeanny ${ }^{1,2,3}$ \\ P Crisanti ${ }^{1,2,3}$ \\ Francine Behar-Cohen ${ }^{1,2,3,4}$ \\ 'INSERM, U872 Physiopathology \\ of ocular diseases:Therapeutic \\ innovations, Paris, France; ${ }^{2}$ Centre de \\ Recherche des Cordeliers, Université \\ Pierre et Marie Curie - Paris6, \\ Paris, France; ${ }^{3}$ Université Paris \\ Descartes, Paris, France; ${ }^{4}$ Department \\ of Ophthalmology, Hôtel-Dieu \\ de Paris, France; ${ }^{5}$ Department of \\ Ophthalmology, Bellevue Hospital, \\ University of Saint-Etienne, France
}

Correspondence: Francine Behar-Cohen Tel +33 ol 40467846 Fax +330I 40467859

Email francine.behar@gmail.com
Purpose: The outer limiting membrane (OLM) is considered to play a role in maintaining the structure of the retina through mechanical strength. However, the observation of junction proteins located at the OLM and its barrier permeability properties may suggest that the OLM may be part of the retinal barrier.

Material and methods: Normal and diabetic rat, monkey, and human retinas were used to analyze junction proteins at the OLM. Proteome analyses were performed using immunohistochemistry on sections and flat-mounted retinas and western blotting on protein extracts obtained from laser microdissection of the photoreceptor layers. Semi-thin and ultrastructure analyses were also reported.

Results: In the rat retina, in the subapical region zonula occludens-1 (ZO-1), junction adhesion molecule (JAM), an atypical protein kinase C, is present and the OLM shows dense labeling of occludin, JAM, and ZO-1. The presence of occludin has been confirmed using western blot analysis of the microdissected OLM region. In diabetic rats, occludin expression is decreased and glial cells junctions are dissociated. In the monkey retina, occludin, JAM, and ZO-1 are also found in the OLM. Junction proteins have a specific distribution around cone photoreceptors and Müller glia. Ultrastructural analyses suggest that structures like tight junctions may exist between retinal glial Müller cells and photoreceptors.

Conclusions: In the OLM, heterotypic junctions contain proteins from both adherent and tight junctions. Their structure suggests that tight junctions may exist in the OLM. Occludin is present in the OLM of the rat and monkey retina and it is decreased in diabetes. The OLM should be considered as part of the retinal barrier that can be disrupted in pathological conditions contributing to fluid accumulation in the macula.

Keywords: retinal barrier, OLM, junctions, adherent, tight, diabetes

\section{Introduction}

Exchanges between the retina and the circulation are controlled by the blood-retinal barrier at the inner side, which is made up of the retinal vessels surrounded by pericytes and glial cells, and by the outer retinal barrier which is constituted by a single layer of retinal pigment epithelial cells (RPE) at the outer side. Tight junctions (TJ) located between endothelial cells in retinal vessels and in retinal pigment epithelial cells contribute to protect the retina towards circulating molecules and cells, to confer immune privilege to the eye, and to limit the penetration of drugs into the retina. Particularly, the RPE cells are responsible for the hydro-ionic exchanges between the choriocapillaries and the outer retina. Junctions in RPE cells are composed, like in other epithelia, by TJ, adherens junctions (AJ) and desmosomes. TJ and AJ differ by 
their constitutional proteins and their spatial organization and their permeability. ${ }^{1,2} \mathrm{TJ}$ are located at the more apical side of the epithelium and are composed of a heterogeneous complex of transmembrane proteins from the occludin, the claudin, and the junctional adhesion molecules (JAM). Intracellular scaffold proteins such as zonula occludens (ZO) link these molecules to the actin cytoskeleton providing structural integrity to the cells ${ }^{3}$ (Figure 1). In the RPE cells, JAM-A and $\mathrm{C}$ are located in the TJ complexes. JAM-A controls apicobasal polarization of the epithelium and regulates its permeability. JAM-C are mostly implicated in the formation of TJ by recruiting the intracellular proteins in the polarization and it allows the basal to apical transmigration of granulocytes. ${ }^{5}$ Directly beneath the TJ are the AJ that consist of the transmembrane protein E-cadherin, and intracellular components, p120-catenin, $\beta$-catenin, and $\alpha$-catenin. Cadherins are transmembrane proteins that link to contact cells and interact with many cytoplasmic proteins. ${ }^{6}$ The AJ performs multiple functions including initiation and stabilization of cell-cell adhesion, regulation of the actin cytoskeleton, intracellular signaling, and transcriptional regulation. More specifically, AJ are required to form and stabilize the TJ through the ZO-1 that can also be a component of AJ and interact with the actin cytoskeleton. ${ }^{7}$ Desmosomes link the AJ to intermediate filaments (Figure 1). Different protein kinase $\mathrm{C}(\mathrm{PKC})$, but more specifically atypical $\mathrm{PKC} \zeta$ such as $\mathrm{PKC} \zeta$ associates and regulates the tight junctions and colocalizes with ZO-1. The assembly of ZO-1 is dependant on $\mathrm{PKC} \zeta$ activity in the mouse embryo. ${ }^{8}$ In endothelial cells that are not linked by $\mathrm{TJ}$, AJ, which is mostly composed

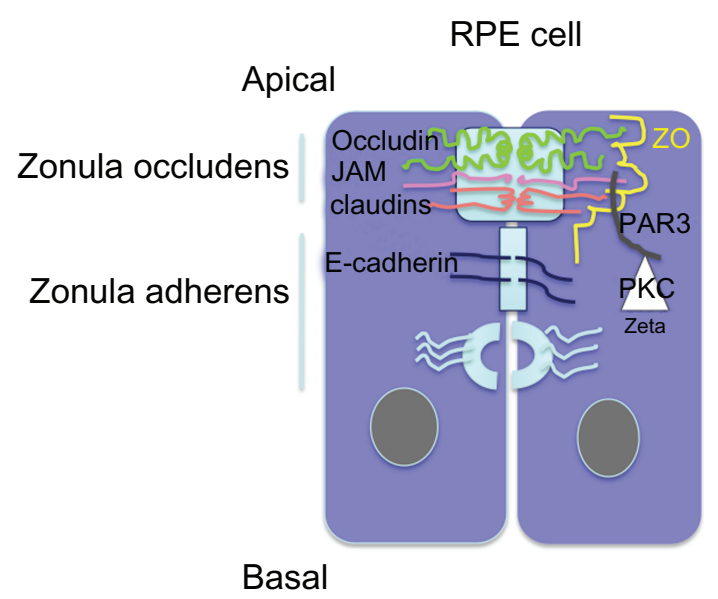

Figure I Schematic representation of apical junction complex (AJC) in epithelial cells. Zonula occludens comprises the tight junctions constituted by members of the transmembrane claudin, occluding, and JAM proteins. Cytoplasmic proteins such as $\mathrm{ZO}$ and $\mathrm{PAR}^{3,6}$ link them to the cytoskeleton. $\mathrm{PKC} \zeta$ phosphorylates these proteins and thereby intervenes in the junction proteins complexes formation.

Abbreviations: JAM, junctional adhesion molecules; PKC, protein kinase C. by VE-cadherin, control the vessel permeability. Vascular endothelial growth factor (VEGF), among other cytokines, induce VE-cadherin phosphorylation and increase vessel permeability and leukocyte diapedesis. ${ }^{9}$

In the vertebrate retina, $\mathrm{AJ}$ and desmosomes have also been identified at the outer limiting membrane (OLM). Indeed, the apical processes of Müller cells are attached to each other and to the inner segments of the photoreceptor cells by continuous heterotypic AJ type that collectively form the OLM. ${ }^{10}$ It has generally been accepted that this "membrane" is continuous throughout the entire retina. However, one observation reported that in the center of the fovea centralis of nonprimate retinas, OLM may contain circumscribed areas in which AJ are lacking between few cone inner segments and apices of Müller cells. ${ }^{10}$ Whether these interruptions also exist in the human fovea remain to be demonstrated. In an unusual role for this type of junction, AJ in the OLM also provide a semipermeable barrier, preventing the diffusion of some proteins out of the extracellular space that surrounds the inner and outer segments of the photoreceptors. The barrier property of the OLM to protein diffusion was demonstrated by subretinal injection of proteins. ${ }^{11}$ In the rabbit retina, the threshold for diffusion was a pore radius estimated between 30 and 36 Angströms. ${ }^{10,12,13}$ Fluorescein isothiocyanate dextran 10-S (radius 22A) diffused readily into the vitreous but the diffusion of FITC dextran 70-S (radius 58A) and 150-S (radius 85) both larger than albumin (radius 35) was markedly slower, and roughly $80 \%$ of the 150-S dextran was still present in the subretinal space after three days. ${ }^{14}$ The semipermeable barrier function of OLM $\mathrm{AJ}$ was related to the unusual and asymmetrical aggregation of actin, myosin, and alpha-actinin in the Müller cells that could also contribute to support the motile photoreceptors. ${ }^{15}$

Interestingly, JAM proteins were recently found in the OLM of mice retinas. Particularly, using immunolocalization studies JAM-C was found at the OLM and at the apical membranes of Müller cells and RPE cells. In JAM-C knock out mice, a normal phenotype was attributed to the expression of JAM-A that could have redundant function. In the all-cone retina of the $\mathrm{Nrl}^{(--)}$mouse, JAM-C was overexpressed suggesting a specific role for JAM proteins in cone polarization and function. ${ }^{16}$

Taken together, these observations suggest that AJ at the OLM may be of a particular type, with components and functions of TJ.

In order to explore this hypothesis, we have used immunohistochemistry of retina sections, western blot analysis from tissue-extracted laser microdissections of specific retinal layers, flat-mounted rat and monkey retinas and semi- and 
ultrathin human macula sections to evaluate the presence of different junction proteins and structures. Moreover, we have evaluated potential changes of the OLM induced by diabetes in one-year diabetic Goto-Kakizaki rats.

\section{Material and methods}

\section{Animals}

The use of animals adhered to the ARVO statement for Ophthalmic and Vision Research and protocols were approved by the ethical committee of René Descartes University of Paris.

To investigate in vivo the effects of diabetes on the OLM, we used Goto-Kakizaki (Male GK Taconic rat) developing spontaneous type 2 diabetes at approximately 14-16 weeks of age. We chose 11 rats GK, 12 months of age, which developed a hyperglycemia ( $>3 \mathrm{mg} / \mathrm{dL}$ ) and 16 normoglycemic rats $(<1.30 \mathrm{mg} / \mathrm{dL})$ as control. Five rats (10 eyes) from each group were used for western blot analysis. The other eye rats were used for immunohistochemistry study on cryosections.

One monkey (1-year-old male Chlorocebus aethiops) was used. One eye was used for cryosections and the other one for retinal flat mounts. We also have used two human eyes from donors to perform semi- and ultrathin analyses.

Animals were sacrificed by $\mathrm{CO}_{2}$ inhalation and the eyes were removed either for cryosections or for retinal flat mounts.

\section{Western blotting}

Western blots were performed on proteins extracted either from the entire neuroretina (at least six eyes from control or diabetic rats) and on proteins extracted from the photoreceptor layers microdissected on cryosections using laser (at least six eyes from control or diabetic rats).

Neuroretinas (10 per group) were homogenized in lysis buffer (10 mM Tris-HCl, pH 7.5, $1 \mathrm{mM}$ EDTA, $1 \mathrm{mM}$ EGTA, $150 \mathrm{mM} \mathrm{NaCl}, 0.5 \%$ Nonidet P40, 1\% Triton X-100, $\beta$-mercaptoethanol) containing protease inhibitor cocktail (from Roche, France). The protein concentration of the resulting lysates was determined using a Bradford assay. $40-50 \mu \mathrm{g}$ of proteins were subjected to SDS-PAGE in a $12 \%$ polyacrylamide gel and electroblotted onto nitrocellulose membranes (Schleicher and Schuell BioScience, Dassel, Germany), anti-glutamine synthetase MAB 302 (Chemicon Technology, Temecula, CA, USA), antioccludin 71-1500 (Zymed, San Francisco, CA, USA), Rat anti $\alpha$-tubulin, clone YL1/2 (Serotec, Düsseldorf, Germany). They were then incubated with corresponding secondary antibodies: peroxidaseconjugated $\mathrm{F}(\mathrm{ab}) 2$ fragment (Caltag, Burlingame, Canada).
Immunoreactive bands were detected with the ECL Western Blotting Detection Reagents Kit (Amersham Biosciences, Orsay, France).

The relative abundance of individual proteins identified was quantified by scanning densitometry.

\section{Laser microdissection}

OLM region was isolated from neuroretina by laser microdissection of outer retina with a Leica AS LMD system (Leica, Solms, Germany) as described by Burbach and colleagues ${ }^{17}$ and protein extracts from at least 30 sections of $40 \mu \mathrm{m}$ per eye were also used for immunoblot analysis.

\section{Immunohistochemistry}

For cryosections, rats or monkey eyes were fixed in 4\% paraformaldehyde in phosphate-buffered saline (PBS), embedded in optimal cutting-temperature (OCT) compound (TissueTek; Miles Inc., Bayer Diagnostics, Puteaux, France) and frozen in liquid nitrogen; we cut $8 \mu \mathrm{m}$ anteroposterior cryosections onto gelatin-coated slides. These slides were stored at $-20{ }^{\circ} \mathrm{C}$ until immunohistochemical analysis. Specimens were incubated overnight at $4{ }^{\circ} \mathrm{C}$ with primary antibodies diluted in PBS supplemented with 10\% fetal calf serum (FCS) and $0.1 \%$ Triton $\mathrm{X}-100$. The primary antibodies used were: anti PKCל (C-20) sc-216 (Santa Cruz Biotechnology, Inc., Santa Cruz, CA, USA), Anti-ZO1 (H-300) sc-10804 (Santa Cruz Biotechnology, Inc.), anti-occludin 71-1500 (Zymed, San Francisco, CA, USA), anti-glutamine synthetase MAB 302 (Chemicon Technology), rhodamine phalloidin R415 (Molecular Probes, Eugene, OR, USA), and Fluorescein Peanut Agglutinin FL-1071 (Vector Laboratories, ABCYS, Paris, France).

After incubation with the primary antibodies, slides were washed with PBS, and incubated with secondary antibodies for one hour at room temperature. Alexa Fluor 488-conjugated goat anti-rabbit $\mathrm{F}(\mathrm{ab}) 2$ fragment or Alexa Fluor 594-conjugated goat anti-mouse F(ab)2 fragment was used as the secondary antibody for immunocytochemistry (Molecular Probes). Sections were incubated with DAPI to stain cell nuclei and were finally observed under a fluorescence microscope (BX51; Olympus, Rungis, France) or a confocal microscope (LSM 510 laser scanning microscope; Zeiss, Le Pecq, France). Each immunostaining was repeated at least three times and staining omitting the primary antibody served as the negative control.

For flat mounts, rats and monkey eyes were enucleated, fixed in 4\% paraformaldehyde (PFA) for 15 minutes at room temperature, and sectioned at the limbus. The cornea and 
lens were discarded. The retinas were carefully peeled from the RPE/choroid/sclera. Retinas were fixed for additional 15 minutes in acetone at $-20{ }^{\circ} \mathrm{C}$ and incubated with the indicated primary and secondary antibodies, each for one hour at room temperature. The retinas were incised, flat mounted, and viewed with a fluorescence microscope.

Serial sections of eyes were mounted on slides. On average, 10-15 sections per eyes were analyzed for each expressed protein that was quantified. Each group had a minimum of five eyes. The experiment was repeated in triplicate. Fluorescent images of sections were scanned at the same magnification and exposure conditions. Quantification was done by Image J software (National Institutes of Health, Bethseda, MD, USA). First, the total area of the section was measured, and then glutamine synthetase (Alexa 594) and occludin (Alexa 488) specific areas were determined on the same section. The percentage of glutamine synthetase- and occludin-specific area was then determined.

\section{Semi- and ultrathin sections}

Enucleated eyes were opened with a blade at the pars plana and immediately fixed in glutaraldehyde for two hours and then dissected with a 6-mm biopsy punch to section the posterior pole. Tissues were fixed in glutaraldhehyde, processed in cacodylate buffer, and embedded in plastic. Semithin sections were obtained using a microtome and counter-stained with hematoxylin-eosin. These sections were analyzed using phase contrast microscopy. Specific sites of interest were further processed for transmission electron microscopy. Ultrathin sections were obtained using an ultramicrotome and counter-stained with uranyl acetate.

\section{Statistical analysis}

Results are expressed as means \pm standard deviation and statistical analysis was performed using a Mann-Whitney $U$ nonparametric test. $P<0.05$ was considered significant.

\section{Results}

We have used different antibodies to label proteins of the tight and adherent junctions as well as glial Müller cells and cone photoreceptors.

\section{Occludin is expressed in the OLM of normal rats and decreased in the OLM of diabetic rats}

As shown in Figure 2, two different zones can be differentiated in the normal rat retina by their differential protein localization (Figures 2A-B). The subapical region of the photoreceptor (SAR) is labeled with the antiphalloidin antibody that recognizes the actin filaments of the cytoskeleton. Indeed while cones are labeled by the peanut agglutinin (PNA) (Figure 2A in green), a well defined organization of actin forms a belt just below the OLM (Figure 2A in red) and photoreceptor inner segments. Interestingly, $\mathrm{PKC} \zeta$, known to control tight-junctions, is also strongly expressed in the subapical region of normal rats (Figure 2B in green).

In the second region, located just below the outer nuclear layer, the apical membranes of Müller cells labeled with glutamine synthetase (Figure 2B in red) also express $\mathrm{PKC} \zeta$ (Figure $2 \mathrm{~B}$ in yellow for colocalization) and form dense areas at the OLM (Figure 2B).

In hyperglycemic diabetic rats, the SAR is altered and the actin belt shows irregularities (Figure 2C). Note that cone photoreceptors are disorganized and that $\mathrm{PKC} \zeta$ expression is decreased and irregularly distributed in the SAR (Figure 2D, arrows).

Controls omitting the first antibodies were negative (Figures 2E and F) demonstrating the specificity of the immunolabeling.

In the OLM region, occludin is expressed and colocalized with glutamine synthetase in condensed areas (Figures 3A, $3 \mathrm{~B}$, and $3 \mathrm{C}$ arrows). Indeed, occludin forms a continuous line at the OLM with some condensed zones (Figure 3B, arrows and inset). This demonstrates that in the normal rat retina, proteins usually known to be expressed in tight junctions are present.

In 1-year-old hyperglycemic GK diabetic rats, occludin expression is decreased, and instead of forming a continuous line, displays focal zones of discontinuities (Figure 3D, arrows). Colabeling of occludin with glutamine synthetase shows that glial Müller cells are swollen and that occludin can be observed in the cytoplasm of the cells suggesting internalization of occludin inside the cells (Figure 3E, inset).

Quantification of fluorescence confirmed that glutamine synthetase (Figure 3F) and occludin expression (Figure 3G) are significantly reduced in the retina from hyperglycemic diabetic rats.

To ascertain occludin expression at the OLM, western blot analyses were performed on protein extracts specifically only from the photoreceptors layers, excluding therefore occludin expressed in endothelial cells. Using this technique, we show that occludin is present in the OLM of normal rat retina and that its expression is significantly decreased in diabetic conditions (Figure 4A).

Moreover, as shown on western blots performed on the entire retina, glutamine synthetase is also significantly 


\section{Normal rat sections}
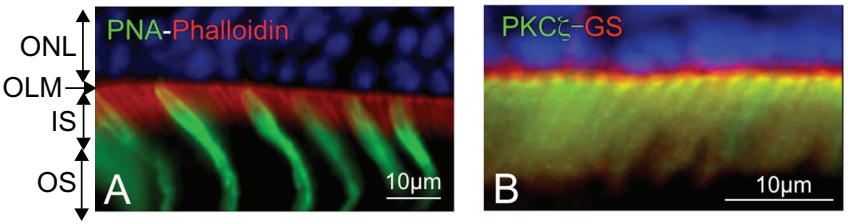

\section{Diabetic rat sections}
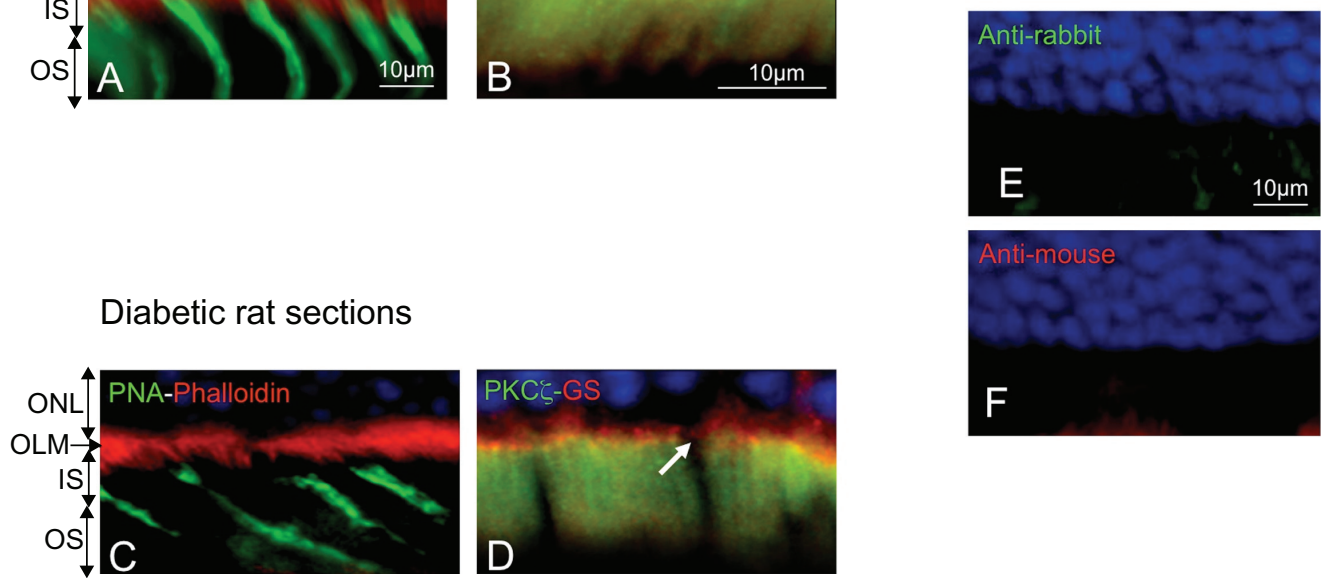

Figure 2 Immunohistochemistry on normoglycemic and hyperglycemic I-year-old Goto-Kakizaki rats. A and C:Phalloidin and peanut agglutinin (PNA) colabeling in normo- or hyperglycemic rat sections. B and D: Atypical PKC $\zeta$ colabeled with glutamine synthetase (GS) a glial Müller marker in normo or hyperglycemic rat sections. E and F: Negative controls omitting the first antibodies. Note the irregular actin (phalloidin staining) and cone photoreceptor disorganization and OLM rupture (arrow) in hyperglycemic conditions (C) and (D).

Notes: In all sections nuclei are stained with DAPI (blue). Bar $=10 \mu \mathrm{m}$ for all sections.

Abbreviations: ONL, outer nuclear layer; OLM, outer limiting membrane; IS, inner segment; OS, outer segment; SAR, subapical region.

Normal rat sections
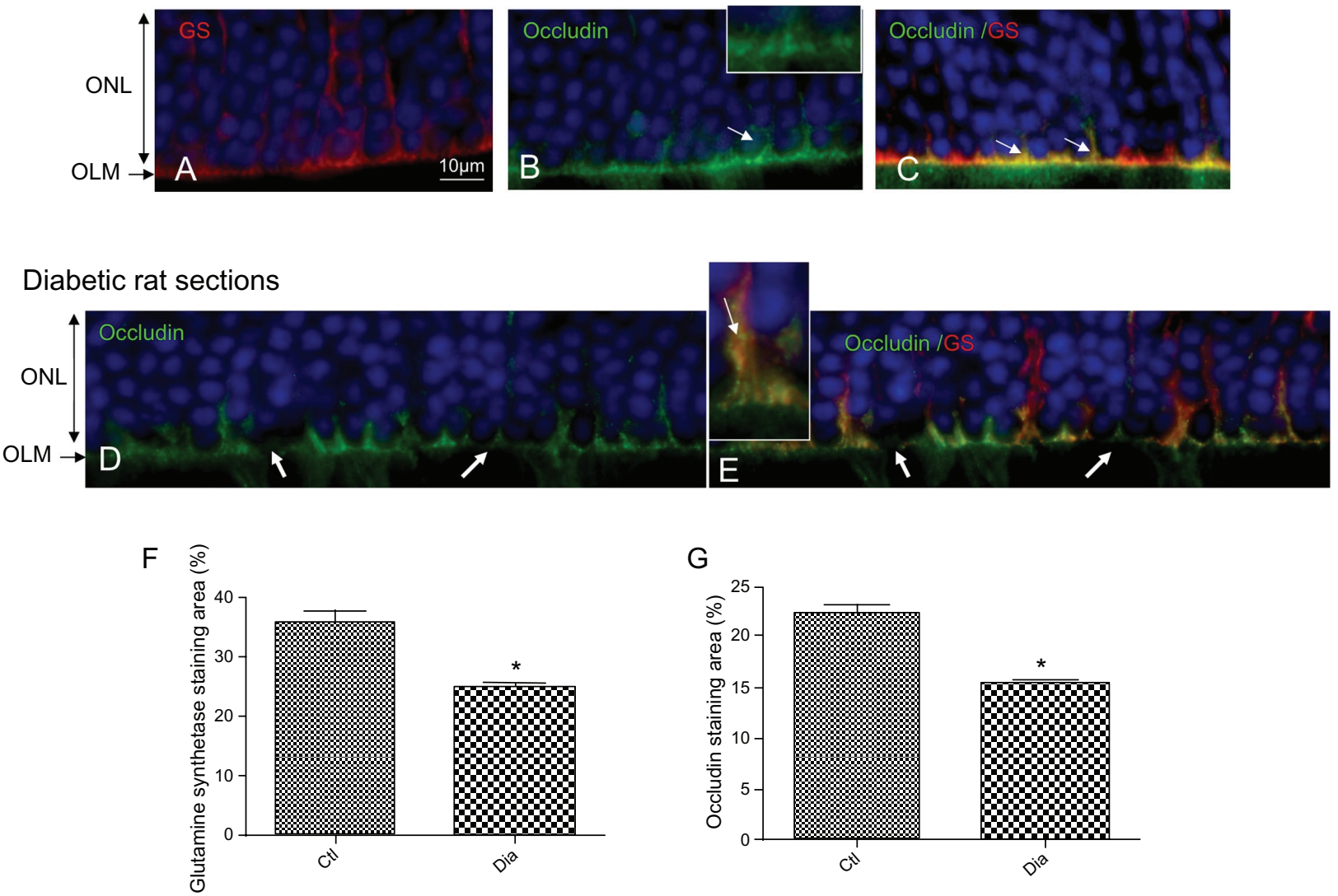

Figure 3 Occludin is expressed and colocalized with glutamine synthetase at the OLM. GS red (A) and occludin green (B) merged (C). Note de decrease of both occludin and GS staining in hyperglycemic conditions (D, E). GS (F) and occludin (G) immunohistochemistry staining quantification.

Abbreviations: ONL, outer nuclear layer; OLM, outer limiting membrane; GS, glutamine synthetase. 
A

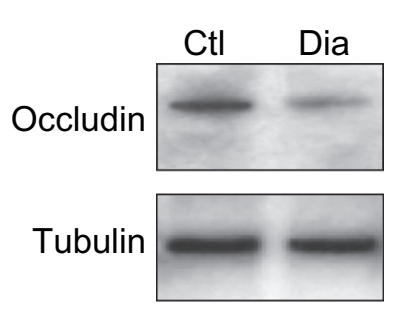

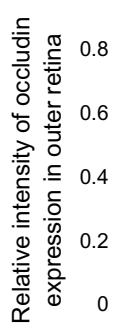

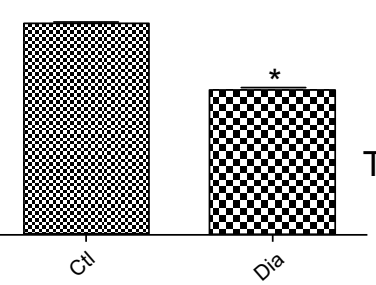

B

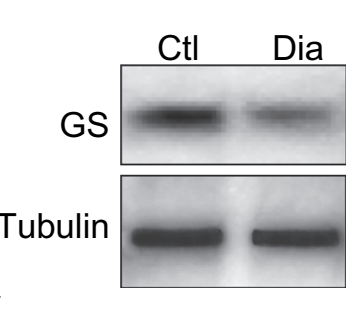

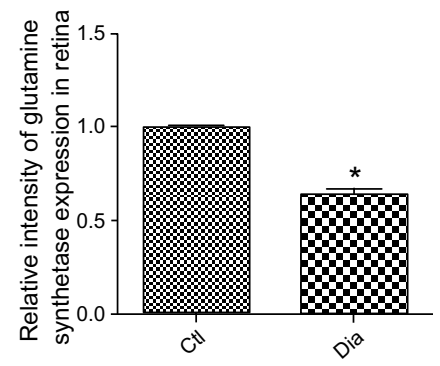

Figure 4 Western blotting analysis and quantification of occludin and GS expression in normal and diabetic conditions. (A) Occludin and tubulin immunoreactivity on protein extracts from OLM region isolated by laser microdissection of outer normal (Ctl) and diabetic (Dia) retina. (B) GS and tubulin immunoreactivity on total protein extracts from normal (Ctl) and diabetic (Dia) retina. These data confirm the expression decrease of both occluding and GS proteins showed by immunochemistry analysis.

Abbreviations: GS, glutamine synthetase; OLM, outer limiting membrane.

decreased in diabetic retina, suggesting the presence of Müller glial cells (Figure 4B).

A closer examination of the OLM region by confocal imaging of flat-mounted retina from normal and diabetic rats showed that the glial Müller cells labeled by glutamine synthetase create a regular and dense network around photoreceptors at the nuclei and subapical level (Figure 5A). Colabeling with glutamine synthetase and occludin confirmed that occludin is located between glial Müller cells and photoreceptors and between glial Müller cells (Figure 5C). Some occludin condensations were observed between glial cells and some photoreceptors (Figure 3C, arrows). In the diabetic rat retinas, a very different pattern of the retinal Müller cells network was observed with swelling of the glial cells forming large cysts (Figure 5B, arrows). Occludin is decreased in the junctions between retinal Müller cells that look open (Figure 5D arrow) and between Müller cells and photoreceptors.

Because the structure of the macula is different from the structure of the rat retina, we examined primate and human retina at the macula.

\section{Junction proteins are expressed in the OLM of the monkey retina}

Contrary to what was previously thought, retinal glial Müller cells are extremely dense in the primate macula where they form a concentric network on flat-mounted retina (Figure 6A). In the fovea, only elongated cones and specific cone-shaped Müller cells are present (Figure 6B). By twodimensional reconstruction of confocal imaging of PNA-GS labeled flat-mounted retina, we could find that indeed cone outer segments are elongated and that the inner segment and the SAR is shorter. Retinal Müller glial cell apices are almost in contact with the cone outer segment leading to a decrease in the glial free SAR that surrounds the fovea (Figure 6C).
Flat mounts of the perifoveal region showed a very dense belt of actin (phalloidin-labeled) in the SAR region just above the cone segments (labeled with PNA in red on Figure 7A). Sections of the monkey retina showed that protein usually known to belong to $\mathrm{TJ}$ are present. $\mathrm{PKC} \zeta$, known to associate with ZO-1 is present and condensed in the OLM and surrounds cone outer segments (Figure 7B, 7C). ZO-1 has a very interesting distribution in the OPL and specifically all around cone photoreceptors as shown by double PNA and ZO-1 labeling. ZO-1 seems to concentrate in the cytoplasma at the SAR region (Figure 7D, arrow) and to localize at the membrane of the cone at the OLM and at the level of cones nuclei (Figure 7D). Colabeling of ZO-1 with GS shows that ZO-1 colocalizes with GS at the OLM and around the cones suggesting that specific junctions may exist at this level (Figure 7E, arrows).

As observed in the rat retina, occludin is expressed in the retinal glial Müller cells at the OLM (Figure 7F, arrows) where it is organized as focal densification between glial apices and the photoreceptors (Figure 7F, inset).

In the monkey retina, JAM-A has a distribution very similar to that of ZO-1. It is found surrounding cones specifically at the OLM and condensed in the cytoplasma at the SAR level (Figure 7G). JAM-A at the OLM colocalizes with glial Müller cells (labeled by GS) at junctions between the apical ends of glial cells and cone photoreceptors (Figure 7G, arrows). All together these results suggest that TJ-like structures may exist in the OLM and that those structures may have differences between glial Müller cells and cones or rod photoreceptors.

\section{Ultrastructural differences in the OLM between the peripheral versus foveal region of the human retina}

In order to evaluate whether specific junction-like structure could also be detected in a human macula, we analyzed 


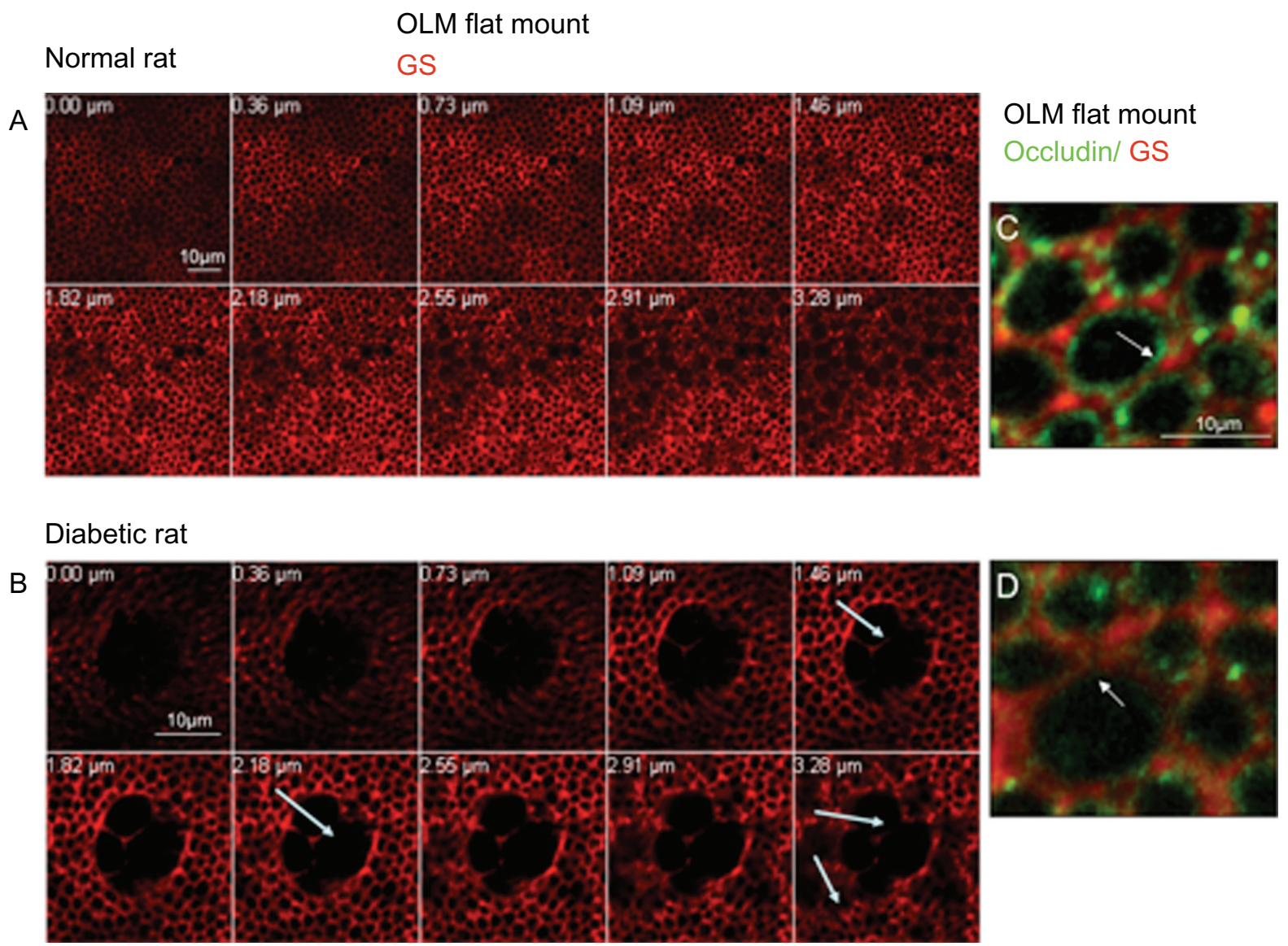

Figure 5 Confocal imaging of the OLM labeled with glutamine synthetase (GS) and occludin. (A) Confocal imaging of the OLM of a normal rat from the SAR towards the ONL (lower panel). (B) Confocal imaging of the OLM of a diabetic rat from the SAR towards the ONL (lower panel), showing large cysts due to glial cells swelling (arrows). (C) Confocal imaging of the OLM labeled with GS and occludin in a normal rat. Occludin is located around photoreceptors at the glial membrane and between glial Müller cells. (D) Confocal imaging of the OLM labeled with GS and occludin in a diabetic rat. The OLM has a very content high of occludin as compared to the normal rat. Junctions seem disrupted between glial Müller cells (arrow).

Abbreviations: GS, glutamine synthetase; OLM, outer limiting membrane; ONL, outer nuclear layer; SAR, subapical layer.

semithin sections of two human maculae (Figure 8A). Very close examination of the macula OLM showed that while a dense continuous line of "junction-like" structure can be observed in the peripheral macula (Figure 8B), this pattern appeared different in the fovea where circumscribed condensed areas could be observed between longer cone outer segments (Figure 8C, black arrows). These areas could correspond to junctions between longer glial Müller apices and the cone outer segments. The scheme on Figure 8D illustrates this hypothesis.

Figure 9 represents ultrathin sections of the equatorial retina of a human eye at the OLM level. Glial Müller cells are joined to each other by homotypic AJ, and are in contact with photoreceptors by heterotypic AJ (Figure 9A, arrows). Higher magnification of the heterotypic AJ showed that while a dense network of actin and intermediate filaments are observed in the glial side of the junction (Figure 9B, arrows), no such networks exist at the photoreceptor side of the junction (Figure 9B, arrowheads). Instead, tight-like junction densifications can be identified (Figure 9B, arrowheads).

In the fovea, junctions between glial Müller cells and photoreceptors in the OLM are shorter and have the typical structure of desmosomes with the dense intermediate filament extensions (Figure 10A, inset). Glial Müller apices send prolongations in between the cones outer segments (Figure 10B, arrow and inset) and tight-like structures can be observed below these glial extensions (Figure 10C, inset).

In epithelial cells, distinct protein localization allows to clearly distinguish between the TJ and the AJ (Figure 11). In the OLM-SAR region, proteins known to be expressed in $\mathrm{TJ}$ are detected and probably assemble differently in the junctions between Müller cells and rods, Müller cells and cones, and between cones and cones. 


\section{Monkey macula}

GS
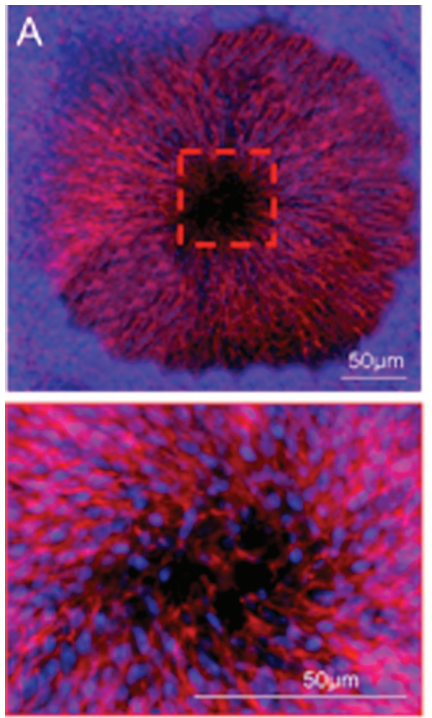

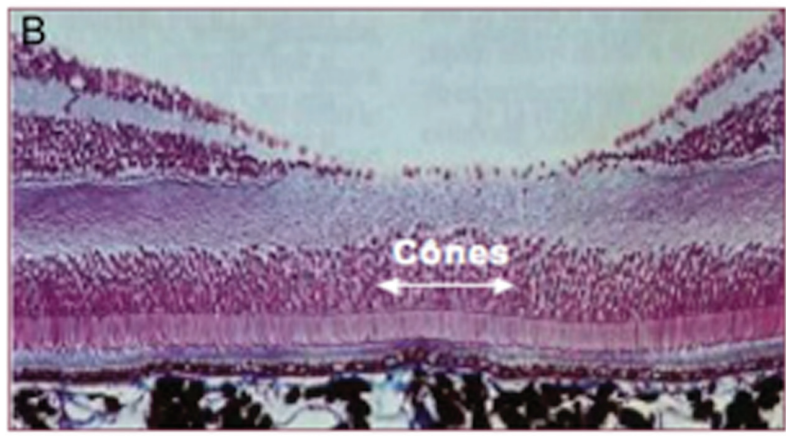

GS/PNA

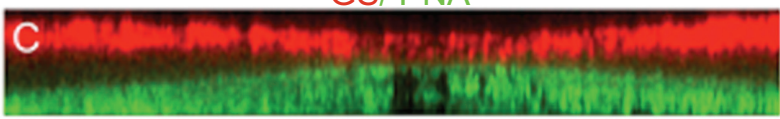

Figure 6 Flat-mounted monkey macula. (A) GS labeling of a flat-mounted macula showing the very dense concentric disposition of glial Müller cells around the fovea centralis. Inset shows a higher magnification of the fovea where glial cells are still present but out of focus. Nuclei are stained in blue. (B, C) Histological section of a monkey macula and computerized reconstitution of GS/PNA-labeled flat-mounted monkey fovea. Outer segments of central cones (green) are longer and in contact with glial Müler cells apical processes (red).

Abbreviation: GS, glutamine synthetase.

\section{Monkey retina}

Phelloidin/ PNA

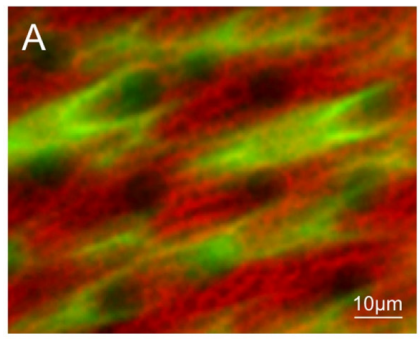

$\mathrm{PKC} \xi$

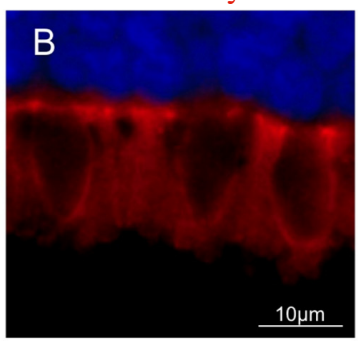

PKC $\xi / P N A$

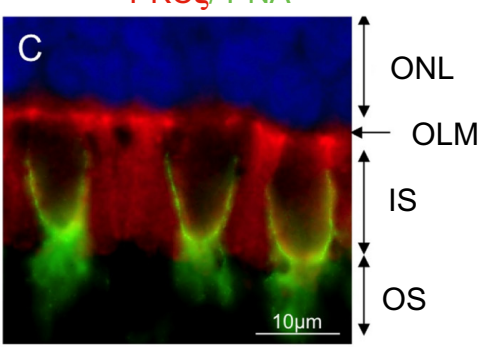

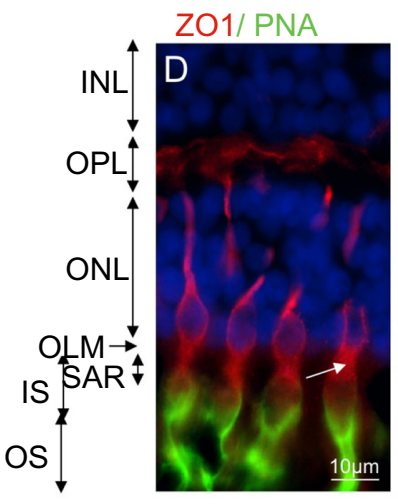
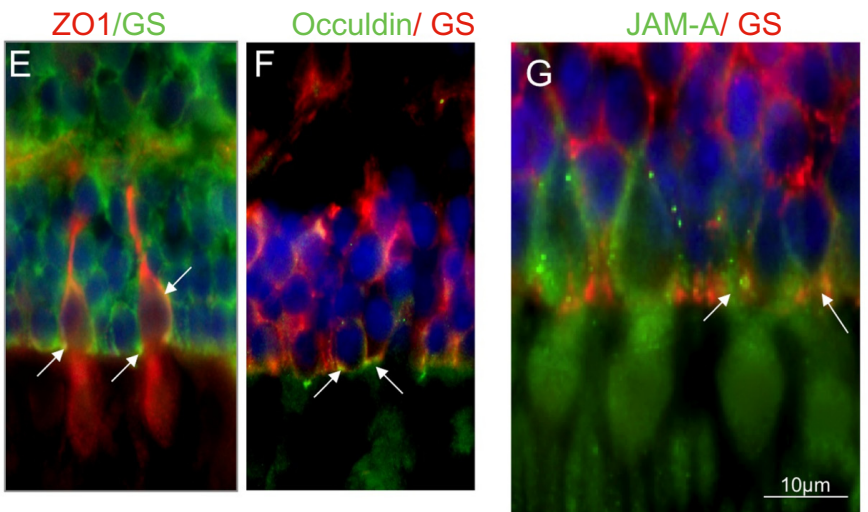

Figure 7 Junction protein immunohistochemistry of the monkey retina. (A) Flat-mounted retina immunostained with phalloidin (actin marker) and peanut agglutinin (cone marker) showing the dense actin belt around photoreceptor SAR. (B) PKC $\zeta$ immunohistochemistry showing that PKC $\zeta$ is localized at the OLM and surrounds cones outer segments. (C) PNA and PKC $\zeta$ co-staining confirms that PKC $\zeta$ surrounds cone outer segments. (D) ZO-I/PNA immunohistochemistry showing that ZO-I surrounding cones are condensed in the SAR and inner segment and are not expressed in rods. (E) ZO-I/GS immunohistochemistry showing that ZO-I is expressed in glial Müller cells membranes around cones and in junctions at the OLM. (F) Occludin/GS immunohistochemistry showing co-staining of occludin in the glial Müller cells apical processes around photoreceptor nuclei in the ONL. (G) JAM-A/GS immunohistochemistry showing that JAM-A follows ZO-I distribution at photoreceptors and Müller glia junctions in the ONL. Notes: Nuclei are stained with DAPI (Blue). Bar $=10 \mu \mathrm{m}$ for all sections.

Abbreviations: ONL, outer nuclear layer; OLM, outer limiting membrane; IS, inner segment; OS, outer segment; SAR, subapical region. 

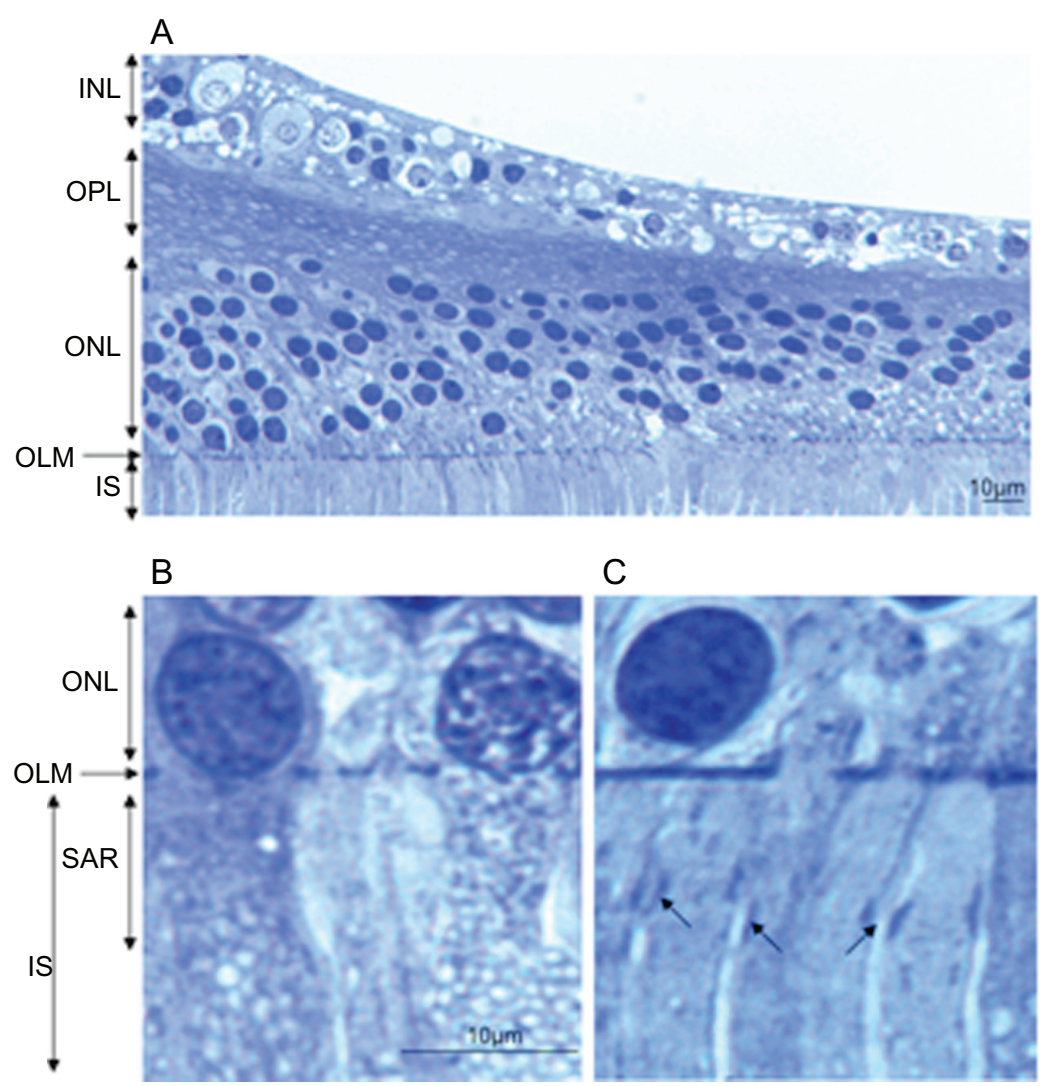

$\mathrm{C}$

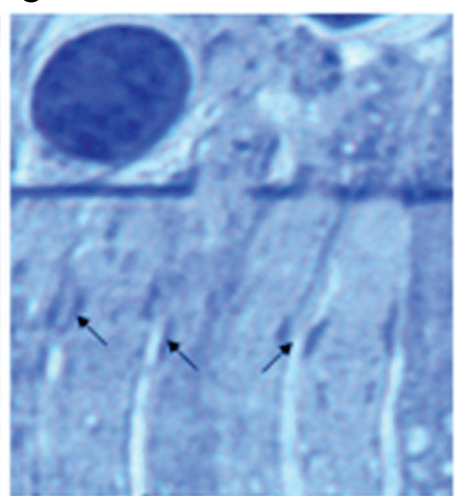

$\mathrm{D}$

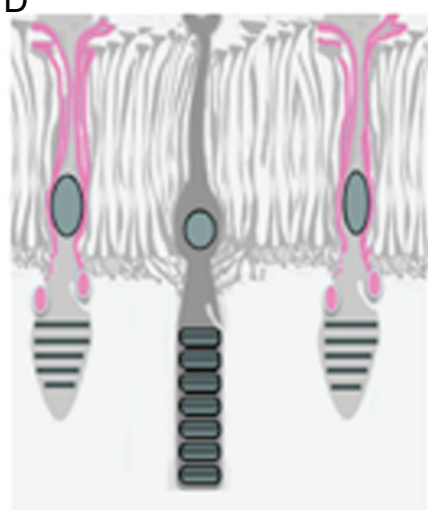

Figure 8 Semithin sections of a human macula. (A) OLM in the macular region. (B) OLM in the peripheral macula. (C) OLM in the fovea. (D) Schematic hypothesis of glial extensions and potential junctions with cone outer segments.

Notes: $\mathrm{Bar}=10 \mu \mathrm{m}$ for all sections.

Abbreviations: ONL, outer nuclear layer; OLM, outer limiting membrane; IS, inner segment; OS, outer segment; SAR, subapical region.

\section{Discussion}

Understanding the exact components of retinal barriers and the specific differences that may exist between the peripheral retina and the macula is a prerequisite for understanding why edema locates in the macula. The OLM is not considered as part of the outer retinal barriers but plays a role in maintaining the structure of the retina through mechanical strength. Whilst the RPE barrier has been well characterized, limited and contradictory data are available regarding the OLM. Moreover, because the structure of the retina differs between species and from the periphery to the macula in humans, no extrapolation can easy be made among species. Several previous studies performed in different species have all shown that when proteins are injected in the subretinal space, depending on their molecular weight, proteins would or would not cross the OLM and diffuse in the inner retina and vitreous, suggesting that the OLM could serve as a gate for macromolecule transport in the retina and act as a semipermeable barrier.

In epithelial cells, apical TJ serve as solute permeability barriers, and AJs have an adhesive role to maintain tissue integrity against mechanical stress. ${ }^{18}$ The TJs and AJs that usually comprise apical junction complexes (AJCs) are widely thought to consist of distinct and largely nonoverlapping sets of proteins. Claudins constitute the major diffusion barrier of $\mathrm{TJ}^{19}$ and contribute to maintain cell polarity with occludin and cytoplasmic proteins such as ZO family member domains in the plasma membrane. ${ }^{20}$ Proteins that constitute the $\mathrm{AJ}$ (mostly cadherins and catenins) do not play a role in the diffusion barrier. ${ }^{20}$

In order to understand the molecular basis of the observed OLM permeability barrier, we have looked for TJ protein expression in the OLM in the rat and the monkey retina. In both species, TJ proteins are expressed and differentially distributed. While actin forms a belt in the SAR of both rods and cones, occludin localizes at the OLM and form a linear pattern around photoreceptors and between glial Müller cells prolongations at the OLM level. As observed on confocal microscopy, occludin is located at the membrane of Müller cells. The mechanisms of occludin assembly to the TJ are still not fully understood. The phosphorylated state of occludin (on Ser/Thr or Tyr residues) by atypical PKC such as PKC $\zeta$ and $\mathrm{PKC} \lambda / \mathrm{l}$ may regulate its interactions with $\mathrm{ZO}-1$ and 

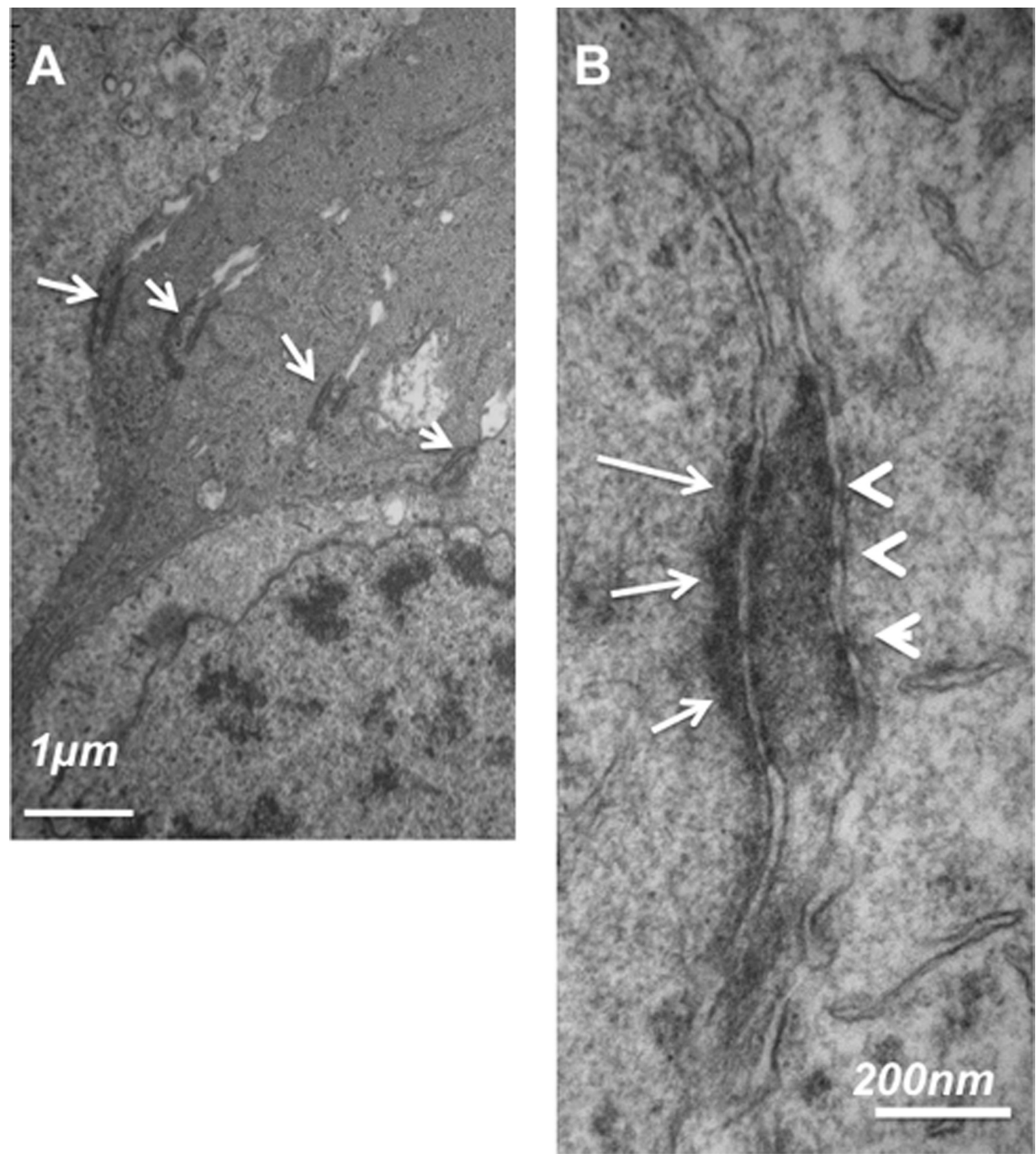

Figure 9 Ultrathin sections of the OLM in the equatorial human retina. (A) junctions at the OLM between glial/glia and glia/photoreceptors. (B) Higher magnification of a heterotypic junction between a Müller glia and a photoreceptor showing asymmetrical disposition of intermediate filaments and tight-like structures (arrowheads). Abbreviation: OLM, outer limiting membrane.

possibly other TJ proteins. ${ }^{21}$ Interestingly, PKC $\zeta$ was found in the SAR of both rods and cones in the rat retina. In the monkey retina, it was also expressed in the SAR of rods and cones but located in dense regions between rods and cones nuclei at the OLM and at the membrane of the cone inner segment. In the retina, $\mathrm{PKC} \zeta$ could assemble with junction proteins or ZO-1 as described in the mouse embryo. ${ }^{22}$ Contrary to ZO-1 previously found in the OLM of mice retina, ${ }^{23}$ occludin was not previously detected in the OLM of the mouse retina. ${ }^{24}$ In the rat and in the monkey retina, using both sections and flat-mounts, occludin was clearly identified at the OLM. These differences can be explained by the different antibodies used and by species differences.

In our experiments, ZO-1 was highly expressed in the rat (not shown) and monkey retina. In the monkey retina, ZO-1 was found in some ganglion cells (not shown) and in the OPL as shown by Puller and colleagues. ${ }^{25}$ In the photoreceptors, ZO-1 was expressed in the cones as shown by the colabeling with PNA, allowing the cone to be followed from its inner segment to the OPL. A denser distribution was observed in the SAR. Most importantly, ZO-1 also was expressed at the junction between glial Müller cells and cones at the OLM, in junction-like structures, where occludin was also localized.

JAM are members of the immunoglobulin superfamily proteins and play a role in paracellular permeability and cell polarity as well as in intracellular signaling regulating cell migration. Three isoforms of similar structure have been described: JAM-A, B, and C. In the mouse retina, JAM-B was recently shown to label an unrecognized class of OFF retinal ganglion cells that responds to upward motion. ${ }^{26} \mathrm{JAM}-\mathrm{C}$ was 

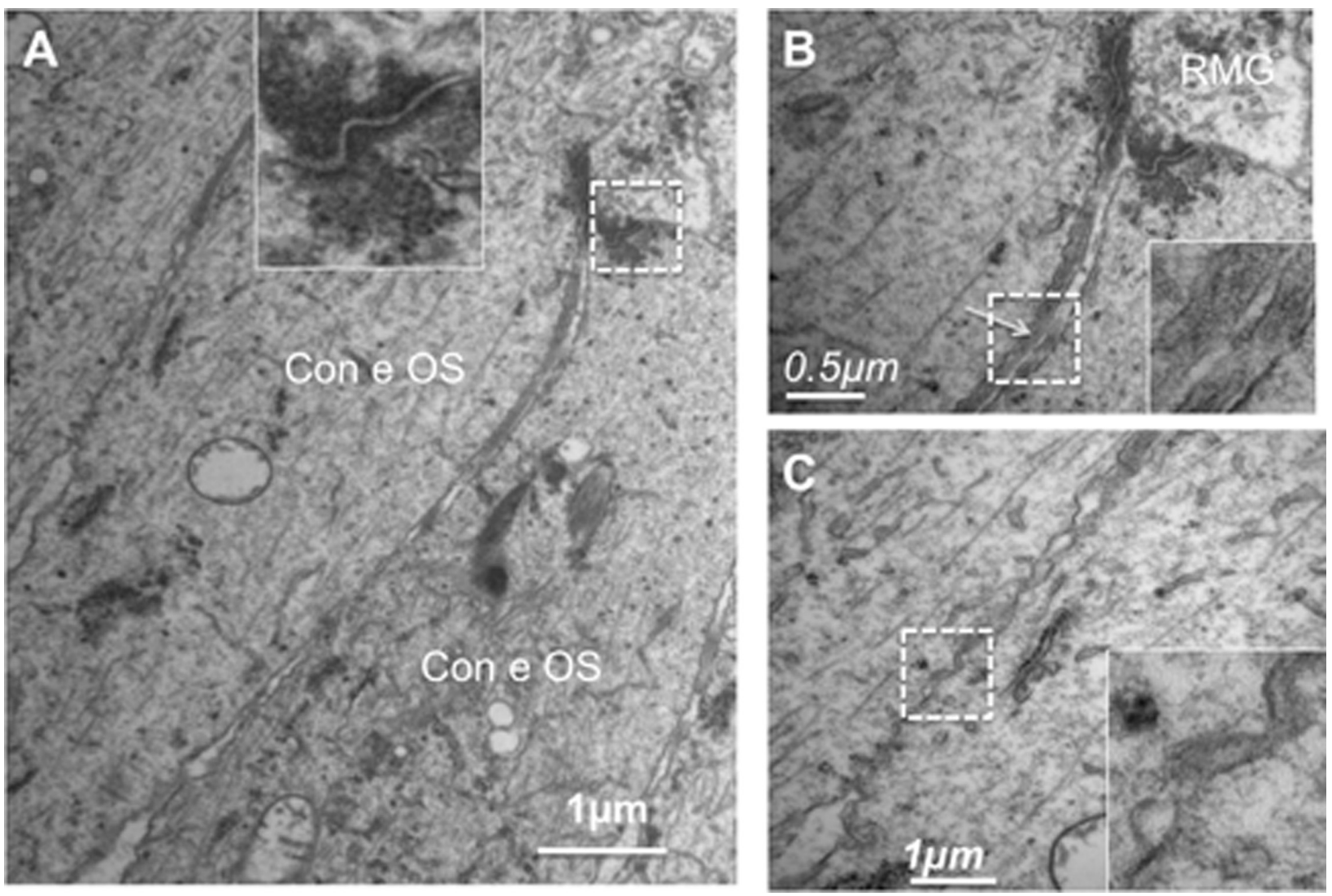

Figure 10 Ultrathin sections of the OLM in the fovea of a human retina. (A) Junctions between Müller glia and cones showing typical desmosome structure (inset) with dense actin filaments. (B) higher magnification of the glial cell extension between cones. (C) Junctions between cones.

Abbreviation: OLM, outer limiting membrane.

found in the OLM of mouse and human retina at the OLM and in the apical processes of retinal Müller glial cells and RPE cells as well as in photoreceptors, which suggests a new role of JAM-C in the interaction between retinal cells. ${ }^{27}$ In JAM-C knock out mice, JAM-A was overexpressed in the OLM. In the monkey retina, JAM-A is highly expressed in the OLM in Müller cell apical processes, in cone photoreceptors pedicles, and in the SAR of both rods and cones. Colabeling with a Müller cell marker (GS) demonstrates that JAM is located in junctions between glial cells and cones in the OLM. The differences between JAM isoforms expressed in the mouse and the monkey retina can be related to species differences. Interestingly, the location of JAM-A found in the monkey retina is very close to the one described for JAM-C in the mouse retina, assuming that these isoforms can have redundant activities.

Ultrastructural analysis of the human retina showed that homotypic and heterotypic junctions can have different structures. Particularly, while we could recognize the well-known AJ structures linking retinal glial Müller cells to each other, a different tight-like structure was observed between Müller glia and photoreceptors. The fact that occludin, ZO-1, and JAM were found at the OLM are in favor of this hypothesis. We also have observed differences in the OLM organization at the fovea, where shorter typical desmosomes linked Müller glia and photoreceptors at the OLM. We also found long Müller glia extension in between cone outer segments and tight-like structures at this level. This observation needs to be confirmed due to the very limited amount of human material available in our study.

Taken together, our results suggest that the OLM can be considered as a tight-like junction structure in the retina with specific recruitments of TJ and AJ proteins (Figure 9). A similar observation was made regarding the junctions between sensory and nonsensory cells of the inner ear where a novel hybrid tight junction with AJ organization has been described..$^{28}$ Our preliminary observations tend to show that the junctions between glial Müller cells and cones may be of a different nature and maybe differently regulated compared to the junctions between Müller cells and rods. Obviously, the different structure of Müller cone cells in the macula and the specific structure of cones suggest that specific molecular link between them and RPE cells villosities may exist. The previous observation that the monkey fovea contains circumscribed zones of disrupted 


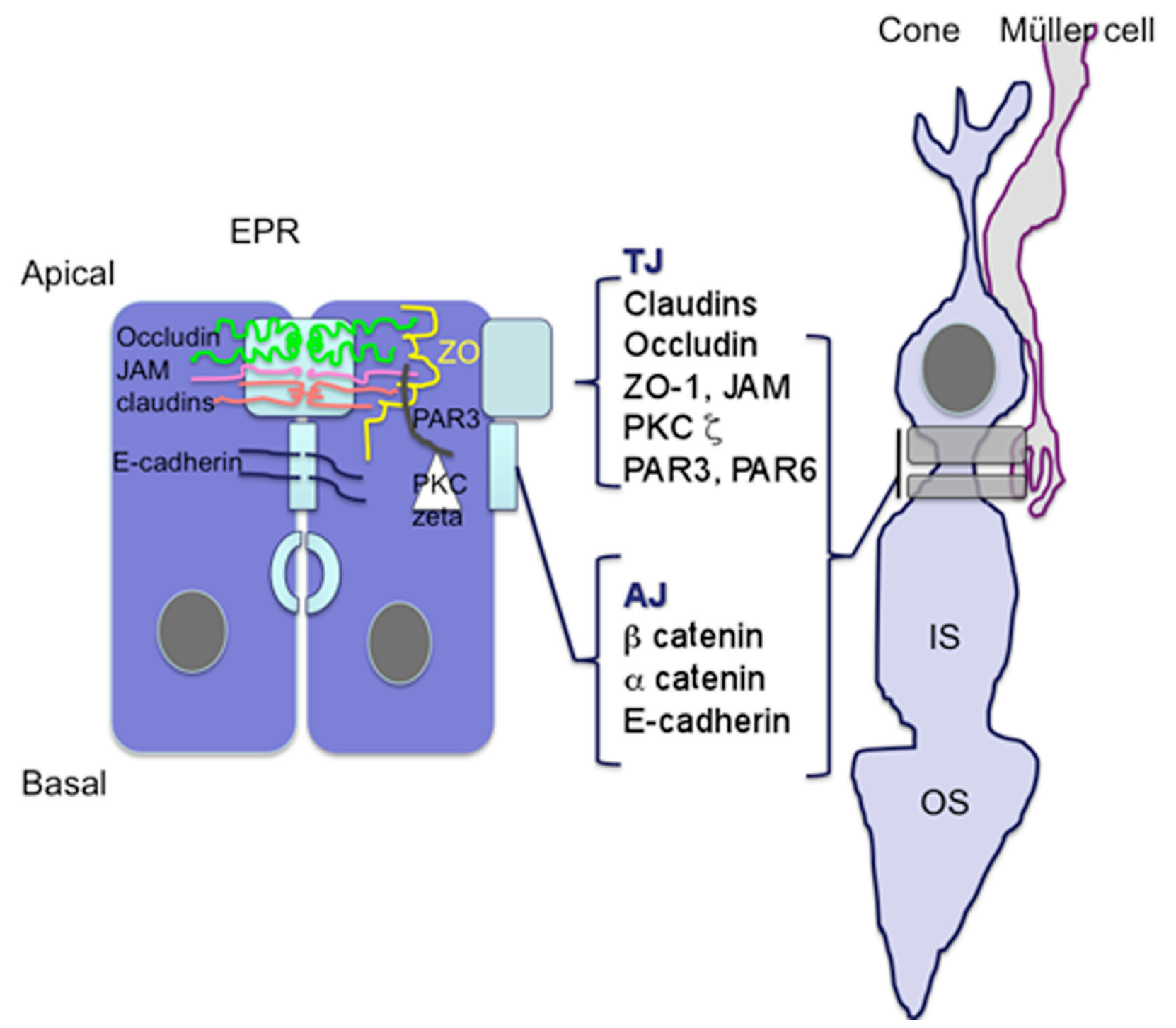

Figure II Schematic representation illustrating that similar to the RPE cell barrier, junctions between Mûller glial cells and photoreceptor cones share common proteins associated with the tight junctions suggesting that OLM could be the third ocular barrier.

Abbreviations: OLM, outer limiting membrane; RPE, retinal pigment epithelium.

OLM may be due to the fact that the junctions in the fovea can be displaced and look shorter. Further close examination of human macula are required to clarify this point.

Finally, we have shown for the first time that during diabetic retinopathy, glial Müller cells are not only swollen but that they lose their occludin content at the OLM level, which leads to cyst formation. These structures are very similar to those described clinically and by optical coherence tomography suggesting that the OLM junctions could be a novel regulatory target in the treatment of macular edema.

In conclusion, our observations are preliminary and will be followed by much more extended analysis of the different $\mathrm{TJ}$ and $\mathrm{AJ}$ proteins in the OLM of different species and particularly of the macula. However, taken together with previous work, ${ }^{27,28}$ they should allow us to rethink the physiopathology of macular edema by taking into account the OLM as a real "third" barrier that can be disrupted during diseases such as diabetic retinopathy.

\section{Acknowledgments}

Drs Crisanti and Behar-Cohen contributed equally to the work. The authors report no conflicts of interest.

\section{References}

1. Tsukita S, Furuse M, Itoh M. Multifunctional strands in tight junctions. Nat Rev. 2001;2:285-293.

2. Koch $\mathrm{S}$, Nusrat J. Dynamic regulation of epithelial cell fate and barrier functions by intercellular junctions. Ann N Y Acad Sci. 2009; 1165:220-227. 
3. Rizzolo LJ. Development and role of tight junctions in the retinal pigment epithelium. Int Rev Cytol. 2007;258:195-234.

4. Mandell KJ, Berglin L, Severson EA, Edelhauser HF, Parkos CA. Expression of JAM-A in the human corneal endothelium and retinal pigment epithelium: localization and evidence for role in barrier function. Invest Ophthalmol Vis Sci. 2007;48:3928-3936.

5. Economopulo M, Hammer J, Wang F, Fariss R, Maminishkis A, Miller SS. Expression, localization and function of JAM-C in human retinal pigment epithelium. Invest Ophthalmol Vis Sci. 2009;50:1454-1463.

6. Chifflet S, Correa V, Nin V, Justet C, Hernández JA. Effect of membrane potential depolarization on the organization of the actin cytoskeleton of eye epithelia. The role of adherens junctions. Exp Eye Res. 2004;79:769-777.

7. Hartsock A, Nelson J. Adherens and tight junctions: Structure, function and connections to the actin cytoskeleton. Biochim Biophys Acta. 2008;778:660-669.

8. Eckert JJ, McCallum A, Mears A, Rumsby MG, Cameron IT, Fleming TP. Relative contribution of cell contact pattern, specific $\mathrm{PKC}$ isoforms and gap junctional communication in tight junction assembly in the mouse early embryo. Dev Biol. 2005;288:234-247.

9. Dejana E, Orsenigo F, Lampugnani MG. The role of adherens junctions and VE-cadherin in thecontrol of vascular permeability. $J$ Cell Sci. 2008;121:2115-2122.

10. Bunt-Milam AH, Saari JC, Klock IB, Garwin GG. Zonulae Adherentes pore size in the external limiting membrane of the rabbit retina. Invest Ophthalmol Vis Sci. 1985;26:1377-1380.

11. Asayama K. In vivo study on the absorption of the subretinal fluid. 2. Studies on an absorption of tracers injected between the sensory retina and the pigment epithelium layer. Acta Soc Ophthalmol Jpn. 1976;80:598-607.

12. Takeuchi A, Kricorian G, Marmor MF. Albumin movement out of the subretinal space after experimental retinal detachment. Invest Ophthalmol Vis Sci. 1995;36:1298-1305.

13. Takeuchi A, Kricorian G, Yao XY, Kenny JW, Marmor MF. The rate and source of albumin entry into soline-filled experimental retinal detachments. Invest Ophthalmol Vis Sci. 1994;35:3792-3798.

14. Marmor MF, Negi A, Maurice DM. Kinetics of macromolecules injected into the subretinal space. Exp Eye Res. 1985;40:687-696.

15. Williams DS, Arikawa K, Paallysaho T. Cytoskeletal components of the adherens junctions between the photoreceptors and the supportive Müller cells. J Comp Neurol. 1990;295:155-164.
16. Daniele LL, Adams RH, Durante DE, Pugh EN Jr, Philp NJ. Novel distribution of junctional adhesion molecule-C in the neural retina and retinal pigment epithelium. J Comp Neurol. 2007;505:166-176.

17. Burbach GJ, Dehn D, Nagel B, Del Turco D, Deller T. Laser microdissection of immunolabeled astrocytes allows quantification of astrocytic gene expression. J Neurosci Methods. 2004;138:141-148.

18. Farquhar MG, Palade GE. Junctional complexes in various epithelia. J Cell Biol. 1963;17:375-412.

19. Furuse M, Sasaki H, Tsukita S. Manner of interaction of heterogeneous claudin species within and between tight junction strands. J Cell Biol. 1999;147:891-903.

20. Tsukita S, Furuse M, Itoh M. Multifunctional strands in tight junctions. Nat Rev Mol Cell Biol. 2001;2:285-293.

21. Rao R. Occludin phosphorylation in regulation of epithelial tight junctions. Ann N Y Acad Sci. 2009;1165:62-68.

22. Eckert JJ, McCallum A, Mears A, Rumsby MG, Cameron IT, Fleming TP Relative contribution of cell contact pattern, specific PKC isoforms and gap junctional communication in tight junction assembly in the mouse early embryo. Dev Biol. 2005;288:234-247.

23. West EL, Pearson RA, Tschernutter M, Sowden JC, Maclaren RE, Ali RR. Pharmacological disruption of the outer limiting membrane leads to increased retinal integration of transplanted photoreceptor precursors. Exp Eye Res. 2008;86:601-611.

24. Paffenholz R, Kuhn C, Grund C, Stehr S, Franke WW. The arm-repeat protein NPRAP (neurojungin) is a constituent of the plaques of the outer limiting zone in the retina, defining a novel type of adhering junction. Exp Cell Res. 1999;250:452-464.

25. Puller C, de Sevilla Müller LP, Janssen-Bienhold U, Haverkamp S. $\mathrm{ZO}-1$ and the spatial organization of gap junctions and glutamate receptors in the outer plexiform layer of the mammalian retina. J Neurosci. 2009;29:6266-6275.

26. Kim IJ, Zhang Y, Yamagata M, Meister M, Sanes JR. Molecular identification of a retinal cell type that responds to upward motion. Nature. 2008;452(7186):478-482.

27. Daniele LL, Adams RH, Durante DE, Pugh EN Jr, Philp NJ. Novel distribution of junctional adhesion molecule-C in the neural retina and retinal pigment epithelium. J Comp Neurol. 2007;505:166-176.

28. Nunes FD, Lopez LN, Lin HW, et al. Distinct subdomain organization and molecular composition of a tight junction with adherens junction features. J Cell Sci. 2006;119(Pt 23):4819-4827.
Clinical Ophthalmology

\section{Publish your work in this journal}

Clinical Ophthalmology is an international, peer-reviewed journal covering all subspecialties within ophthalmology. Key topics include: Optometry; Visual science; Pharmacology and drug therapy in eye diseases; Basic Sciences; Primary and Secondary eye care; Patient Safety and Quality of Care Improvements. This journal is indexed on

\section{Dovepress}

PubMed Central and CAS, and is the official journal of The Society of Clinical Ophthalmology (SCO). The manuscript management system is completely online and includes a very quick and fair peer-review system, which is all easy to use. Visit http://www.dovepress.com/ testimonials.php to read real quotes from published authors. 